QUARTERLY OF APPLIED MATHEMATICS

VOLUME LXIV, NUMBER 1

MARCH 2006, PAGES 137-151

$\mathrm{S} 0033-569 \mathrm{X}(06) 00995-\mathrm{X}$

Article electronically published on January 24, 2006

\title{
ON STABILITY OF LINEAR TIME-VARYING SECOND-ORDER DIFFERENTIAL EQUATIONS
}

BY

LUU HOANG DUC (Fachbereich Mathematik, J.W. Goethe Universität, Frankfurt, Germany),

ACHIM ILCHMANN (Institut für Mathematik, Technische Universität Ilmenau, Ilmenau, Germany),

STEFAN SIEGMUND (Fachbereich Mathematik, J.W. Goethe Universität, Frankfurt, Germany),

AND

PETER TARABA (Fachbereich Mathematik, J.W. Goethe Universität, Frankfurt, Germany)

Abstract. We derive sufficient conditions for stability and asymptotic stability of second order, scalar differential equations with differentiable coefficients.

1. Introduction. We study, for differentiable $a_{0}, a_{1}: \mathbb{R}_{\geq 0} \rightarrow \mathbb{R}$, stability properties of linear time-varying second-order differential equations of the form

$$
\ddot{x}+a_{1}(t) \dot{x}+a_{0}(t) x=0,
$$

which describe, if $a_{0}, a_{1}$ are non-negative, an oscillator with damping $a_{1}$ and rigidity $a_{0}$.

Numerous sufficient conditions for uniform (and asymptotic) stability of (1.1) are derived in terms of bounds of the coefficients and its derivatives. These results are presented in Section 2, In the remainder of the present section, we first recall and make precise various concepts of stability, and then give a brief overview about the results available in the literature and related to our findings.

Writing $y=\left(y_{1}, y_{2}\right)^{T}=(x, \dot{x})^{T}$, equation (1.1) is equivalent to the planar first-order system

$$
\dot{y}=A(t) y, \quad \text { where } \quad A(t)=\left[\begin{array}{cc}
0 & 1 \\
-a_{0}(t) & -a_{1}(t)
\end{array}\right] .
$$

For given $\left(x^{0}, x^{1}\right)^{T}=y^{0} \in \mathbb{R}^{2}$ and $t_{0} \geq 0$, we denote by $x\left(\cdot ; t_{0}, x^{0}, x^{1}\right): \mathbb{R}_{\geq 0} \rightarrow \mathbb{R}$, $y\left(\cdot ; t_{0}, y^{0}\right): \mathbb{R}_{\geq 0} \rightarrow \mathbb{R}^{2}$ the solution of (1.1), (1.2) with initial data

$$
\left(x\left(t_{0} ; t_{0}, x^{0}, x^{1}\right), \dot{x}\left(t_{0} ; t_{0}, x^{0}, x^{1}\right)\right)=\left(x^{0}, x^{1}\right), \quad y\left(t_{0} ; t_{0}, y^{0}\right)=y^{0},
$$

Received April 11, 2005.

2000 Mathematics Subject Classification. Primary 34A30, 34D20, 35B40 .

E-mail address: Ihduc@math.uni-frankfurt.de

E-mail address: achim.ilchmann@tu-ilmenau.de

E-mail address: siegmund@math.uni-frankfurt.de

E-mail address: taraba@math.uni-frankfurt.de 
respectively. Uniqueness and existence of the solution on $\mathbb{R}_{\geq 0}$ is well known.

To investigate stability properties of the zero solution of (1.1), respectively of (1.2), or sufficient conditions of it, we recall the following definitions:

Definition 1.1. The zero solution of (1.2), and equally (1.1), is said to be

- stable if, and only if, for every $t_{0} \geq 0$ and $\varepsilon>0$ there exists a $\delta=\delta\left(\varepsilon, t_{0}\right)>0$ such that

$$
\left\|y^{0}\right\|<\delta \quad \Longrightarrow \quad\left\|y\left(t ; t_{0}, y^{0}\right)\right\|<\varepsilon \quad \text { for all } t \geq t_{0} ;
$$

- uniformly stable if, and only if, it is stable and the $\delta$ in (i) does not depend on $t_{0}$

- attractive if, and only if, for all $\left(y^{0}, t_{0}\right) \in \mathbb{R}^{2} \times \mathbb{R}_{\geq 0}$, we have $\lim _{t \rightarrow \infty} y\left(t ; t_{0}, y^{0}\right)=0$;

- uniformly attractive if, and only if, for all $\delta>0$ there exists $T=T(\delta)>0$ such that, for all $t_{0} \geq 0$, for all $t \geq t_{0}+T$, and for all $y^{0} \in \mathbb{R}^{2}$ we have $\left\|y\left(t ; t_{0}, y^{0}\right)\right\| \leq \delta\left\|y^{0}\right\|$

- (uniformly) asymptotically stable if, and only if, it is (uniformly) stable and (uniformly) attractive, respectively.

REMARK 1.2.

(a) Stability, (uniform) asymptotic stability and (uniform) attractivity can be defined for arbitrary solutions of system (1.2). By linearity of system (1.2) each solution has the same stability type and hence we may speak of stability properties of the system instead of stability properties of the zero solution.

(b) We repeatedly use the fact that for linear systems attractivity implies stability, i.e. if system (1.2) is attractive, then it is already asymptotically stable.

(c) To prove stability, asymptotic stability or attractivity it suffices to check the conditions in Definition 1.1 for a single $t_{0}^{*} \geq 0$ instead of for all $t_{0} \geq 0$, since one can use continuous dependence of the solution on the initial condition to estimate the size of $\left\|\lambda\left(t ; t_{0}, y^{0}\right)\right\|$ on the compact interval $\left[\min \left\{t_{0}, t_{0}^{*}\right\}, \max \left\{t_{0}, t_{0}^{*}\right\}\right]$.

A first step in the direction of sufficient conditions for asymptotic stability is via the time depending eigenvalues of the matrix $A(t)$ in (1.2). However, in general these eigenvalues do not contain sufficient or necessary information about the stability type; Hoppenstead [7] gives a two-dimensional example where both eigenvalues of $A(t)$ are located at -1 for all $t \geq 0$, however, the system has an unstable solution. Only if the eigenvalues of $A(t)$ depend "slowly" on time, then asymptotic stability is related to the time-dependent eigenvalues; see for example Desoer [3], Rosenbrock [14], Solo [19], Rugh [15] and the references in the latter textbook.

A different approach using topological methods is the Sacker-Sell or dichotomy spectrum (see Siegmund [17]). The spectrum consists of so-called spectral intervals in $\mathbb{R}$ which generalize the eigenvalue real parts to the nonautonomous situation. If the spectrum is negative, system (1.2) is uniformly asymptotically stable. Since only in rare cases is it possible to compute the dichotomy spectrum directly from the coefficients $a_{0}$ and $a_{1}$, we do not follow this approach but instead derive stability criteria based on the coefficients.

If the rigidity coefficient $a_{0}(\cdot) \equiv a_{0}>0$ is constant and positive, then many sufficient conditions for stability and attractivity of the zero solution of (1.1) are known. 
One approach to derive asymptotic stability of (1.1) is via an appropriate Lyapunovfunction and by invoking LaSalle's Invariance Principle. If the coefficients of (1.1) satisfy

$$
a_{0}(\cdot) \equiv a_{0}>0 \quad \text { and } \quad a_{1}(t) \geq 0 \quad \forall t \geq 0,
$$

then the derivative of $V(t):=\dot{x}(t)^{2}+a_{0} x(t)^{2}$ along the solution $x(\cdot) \equiv x\left(\cdot ; t_{0}, x^{0}, x^{1}\right)$, $\left(x^{0}, x^{1}\right) \in \mathbb{R} \times \mathbb{R}$, yields, for all $t_{0} \geq 0$,

$$
\frac{\mathrm{d}}{\mathrm{d} t} V(t)=-2 a_{1}(t) \dot{x}(t)^{2} \leq 0, \quad \forall t \geq t_{0},
$$

whence the zero solution is uniformly stable. Moreover, if

$$
\exists \underline{a}_{1}, \bar{a}_{1}>0 \quad \forall t \geq 0: 0<\underline{a}_{1} \leq a_{1}(t) \leq \bar{a}_{1},
$$

holds, then, for all $t \geq 0, \frac{\mathrm{d}}{\mathrm{d} t} V(t) \leq-2 \underline{a}_{1} \dot{x}(t)^{2}$. Therefore, LaSalle's Invariance Principle yields that $\lim _{t \rightarrow \infty} \dot{x}(t)=0$, the $\omega$-limit set of the solution is $\ddot{x}+a_{0} x=0$, and hence that $\lim _{t \rightarrow \infty} x(t)=\lim _{t \rightarrow \infty} \dot{x}(t)=0$. This result is well known (see Theorem 1 in [10]) and was also stated in [1]:

Theorem 1.3 (Levin and Nohel [10]). If the coefficients of (1.1) satisfy (1.3) and (1.4), then the zero solution of (1.1) is asymptotically stable.

The upper bound in condition (1.4) cannot be omitted, as can be seen from the equation

$$
\ddot{x}+\left(1+a_{0}+e^{t}\right) \dot{x}+a_{0} x=0
$$

with positive and constant $a_{0}>0$, since it admits the solution $t \mapsto x(t)=1 / a_{0}+e^{-t}$, and therefore the zero solution is not attractive.

In an attempt to weaken condition (1.4) two cases have been distinguished:

$$
\text { small damping: } a_{0}(\cdot) \equiv a_{0}>0 \text { and } \exists \bar{a}_{1}>0 \forall t \geq 0: 0 \leq a_{1}(t) \leq \bar{a}_{1}
$$

and

$$
\text { large damping: } a_{0}(\cdot) \equiv a_{0}>0 \quad \text { and } \quad \exists \underline{a}_{1}>0 \forall t \geq 0: 0<\underline{a}_{1} \leq a_{1}(t) .
$$

In both cases, asymptotic stability is not guaranteed: for small damping there might be an oscillation so that the zero solution is not attractive; for large damping one has to exclude the phenomenon of overdamping, i.e. the existence of a solution $x$ with $\lim _{t \rightarrow \infty} x(t)=x_{*} \neq 0$. An example of the latter is equation (1.5).

Smith [18] proved that the condition

$$
A_{1}(t):=\int_{0}^{t} a_{1}(s) d s \rightarrow \infty \quad \text { as } t \rightarrow \infty
$$

is necessary for the asymptotic stability of equation (1.1). For the case of large damping he even gave a necessary and sufficient condition.

Theorem 1.4 (Smith [18). Suppose the coefficients of equation (1.1) satisfy large damping. Then the condition

$$
\int_{0}^{\infty} e^{-A_{1}(t)} \int_{0}^{t} e^{A_{1}(s)} d s d t=\infty
$$

is equivalent to asymptotic stability of the zero solution of equation (1.1). 
Hatvani, Krisztin and Totik [6] proved that for any $c>0$ condition (1.6) is equivalent to

$$
\sum_{n=1}^{\infty}\left[A_{1}(n c)^{-1}-A_{1}((n-1) c)^{-1}\right]^{2}=\infty
$$

which is sometimes easier to check, as can be seen from the examples $a_{1}(t)=t$ which does satisfy condition (1.6) and $a_{1}(t)=t^{2}$ which does not. In general condition (1.7) is still difficult to verify. The following two theorems provide sufficient conditions in the cases of large and small damping.

Theorem 1.5 (Artstein, Infante [1]). Suppose the coefficients of equation (1.1) satisfy large damping and, in addition, for some constants $A, B>0$,

$$
\int_{0}^{t} a_{1}(s) d s \leq A+B t^{2} \quad \text { for all } t \geq 0 .
$$

Then the zero solution of (1.1) is attractive and therefore asymptotically stable. Moreover, the exponent 2 is optimal in the sense that it cannot be replaced by any $2+\varepsilon$, $\varepsilon>0$.

Theorem 1.6 (Hatvani [5]). In the case of small damping,

$$
\limsup _{t \rightarrow \infty}\left(t^{-2 / 3} \int_{0}^{t} a_{1}(s) d s\right)>0
$$

is sufficient for asymptotic stability of equation (1.1). Moreover, the exponent $-2 / 3$ is optimal in the sense that it cannot be replaced by any $\varepsilon-2 / 3, \varepsilon>0$.

There are many results on asymptotic stability of certain nonlinear equations. We merely mention a series of papers by Pucci and Serrin [11, 12, 13]; see also the references therein. A typical result restricted to the linear case (1.1) is the following theorem.

Theorem 1.7 (Pucci, Serrin [11]). Suppose there exist continuous functions $\sigma, \delta: \mathbb{R}_{\geq 0} \rightarrow$ $\mathbb{R}_{\geq 0}$ so that $\sigma \delta$ is bounded but

$$
\int_{0}^{t} \sigma(\tau) \mathrm{d} \tau \rightarrow \infty \quad \text { as } \quad t \rightarrow \infty
$$

and either $\sigma$ is of bounded variation or $\log \sigma$ is uniformly Lipschitz on $\mathbb{R}_{\geq 0}$.

If the coefficients of (1.1) satisfy

$$
a_{0}(\cdot) \equiv a_{0}>0 \quad \text { and } \quad 0 \leq \sigma(t) \leq a_{1}(t) \leq \delta(t) \quad \forall t \geq 0,
$$

then the zero solution of (1.1) is asymptotically stable.

Compared to the amount of literature on small and large damping, there are only a few results on the stability of (1.1) available, where both coefficients $a_{0}(\cdot)$ and $a_{1}(\cdot)$ are allowed to be non-constant; see, e.g. Ignatyev [9] and, for the case of complex-valued coefficient functions, Hovhannisyan [8] and the references therein.

Theorem 1.8 (Ignatyev 9]). Suppose that the coefficients of (1.1) satisfy the following:

$$
\begin{aligned}
\exists M>0 \forall t \geq 0: & \left|\dot{a}_{0}(t)\right|+\left|a_{1}(t)\right| \leq M, \\
\exists \underline{a}>0 \forall t \geq 0: & 0<\underline{a} \leq \dot{a}_{0}(t)+2 a_{0}(t) a_{1}(t) .
\end{aligned}
$$


Then the zero solution of (1.1) is uniformly asymptotically stable.

2. Criteria for asymptotic stability. In this section we extend some of the results mentioned in the Introduction. The statement of the first theorem is in the spirit of Theorem 1.8, but the uniform bound on the absolute value of $\dot{a}_{0}$ is not needed. However, the method of proof is different, and we only show uniform stability and attractivity, not uniform asymptotic stability.

Theorem 2.1. Suppose that the coefficients of (1.1) satisfy the following

$$
\begin{array}{rll}
\exists \underline{a}>0 \forall t \geq 0: & 0<\underline{a}_{0} \leq a_{0}(t) \leq \bar{a}_{0}, \\
\exists \bar{a}_{1}>0 \forall t \geq 0: & \left|a_{1}(t)\right| \leq \bar{a}_{1}, \\
\exists \underline{a}>0 \forall t \geq 0: & 0<\underline{a} \leq \dot{a}_{0}(t)+2 a_{0}(t) a_{1}(t) .
\end{array}
$$

Then the zero solution of (1.1) is asymptotically stable. In fact, it is attractive and uniformly stable.

REMARK 2.2. If the rigidity coefficient $a_{0}(\cdot) \equiv a_{0}$ is a positive constant, then conditions (2.1)-(2.3) specialize to

$$
0<\frac{\underline{a}}{2 a_{0}} \leq a_{1}(t) \leq \bar{a}_{1} \quad \forall t \geq 0
$$

and therefore Theorem 2.1 generalizes Theorem 1.3

Proof of Theorem 2.1. Let $\left(x^{0}, x^{1}\right) \in \mathbb{R} \times \mathbb{R}$, and let $x(\cdot) \equiv x\left(\cdot ; 0, x^{0}, x^{1}\right)$ be the solution to (1.1) starting at time 0 in $\left(x^{0}, x^{1}\right)$. In view of (2.1) and (2.3), the derivative of

$$
V(t):=\frac{1}{2} x(t)^{2}+\frac{1}{2 a_{0}(t)} \dot{x}(t)^{2}, \quad t \geq 0,
$$

along the solutions of (1.1) satisfies, by omitting the argument $t$,

$$
\begin{aligned}
\dot{V}(t)=x \dot{x}+\frac{1}{a_{0}} \dot{x} \ddot{x}-\frac{\dot{a}_{0}}{2 a_{0}^{2}} \dot{x}^{2}=x \dot{x}+\frac{1}{a_{0}} \dot{x}\left(-a_{1} \dot{x}-a_{0} x\right)-\frac{\dot{a}_{0}}{2 a_{0}^{2}} \dot{x}^{2} & \\
& =-\frac{1}{2 a_{0}^{2}}\left(\dot{a}_{0}+2 a_{0} a_{1}\right) \dot{x}^{2} \leq-\frac{a}{2 \bar{a}_{0}^{2}} \dot{x}^{2} \leq 0,
\end{aligned}
$$

and so, by integration and invoking (2.1),

$$
\frac{1}{2} x(t)^{2}+\frac{1}{2 \bar{a}_{0}} \dot{x}(t)^{2} \leq V(t) \leq V(0) \leq \frac{1}{2}\left(x^{0}\right)^{2}+\frac{1}{2 \underline{a}_{0}}\left(x^{1}\right)^{2} \quad \forall t \geq 0 .
$$

Therefore, the zero solution of (1.1) is uniformly stable.

Note also that, in view of (2.1) and (2.2), $\ddot{x}=-a_{1} \dot{x}-a_{0} x$ is bounded. Furthermore,

$$
V(0) \geq V(0)-V(t)=-\int_{0}^{t} \dot{V}(\tau) \mathrm{d} \tau \geq \frac{\underline{a}}{2 \bar{a}_{0}^{2}} \int_{0}^{t} \dot{x}(\tau)^{2} \mathrm{~d} \tau \quad \forall t \geq 0,
$$

and so $\dot{x}$ is square integrable. Finally, since $\dot{x}$ is square integrable and uniform continuous, the latter is a consequence of boundedness of $\ddot{x}$, and we may apply Barbălat's lemma [2], which then yields $\lim _{t \rightarrow \infty} \dot{x}(t)=0$.

Next we prove that $V(t) \rightarrow 0$ as $t \rightarrow \infty$, which then implies that $x(t) \rightarrow 0$ as $t \rightarrow \infty$. Supposing that this is not true, then due to the fact that $t \mapsto V(t)$ is monotonically 
non-increasing, there exists $\delta>0$ such that $V(t) \geq \delta^{2}$ for all $t \geq 0$. Since $\dot{x}(t) \rightarrow 0$ as $t \rightarrow \infty$, there exists $t_{1} \geq 0$ such that

$$
x(t)^{2}=2 V(t)-\frac{1}{a_{0}(t)} \dot{x}(t)^{2} \geq 2 V(t)-\frac{1}{\underline{a}_{0}} \dot{x}(t)^{2} \geq \delta^{2} \quad \forall t \geq t_{1} .
$$

By continuity of $x$, this means that either $x(t) \geq \delta$ or $x(t) \leq-\delta$ for all $t \geq t_{1}$. Suppose that $x(t) \geq \delta$ for all $t \geq t_{1}$.

Then $\ddot{x}(t)=-a_{1}(t) \dot{x}(t)-a_{0}(t) x(t) \leq\left|\bar{a}_{1}\right||\dot{x}(t)|-\underline{a}_{0} \delta$, together with $\dot{x}(t) \rightarrow 0$ as $t \rightarrow \infty$, yields existence of $t_{2} \geq t_{1}$ such that

$$
\ddot{x}(t) \leq-\underline{a}_{0} \frac{\delta}{2} \quad \forall t \geq t_{2} .
$$

Integration gives

$$
\dot{x}(t)-\dot{x}\left(t_{2}\right)=\int_{t_{2}}^{t} \ddot{x}(\tau) \mathrm{d} \tau \leq-\underline{a}_{0} \frac{\delta}{2}\left(t-t_{2}\right) \quad \forall t \geq t_{2},
$$

and, by repeated integration, we arrive at

$$
\begin{aligned}
\delta-x\left(t_{2}\right) \leq x(t)-x\left(t_{2}\right)=\int_{t_{2}}^{t} \dot{x}(\tau) \mathrm{d} \tau & \leq \int_{t_{2}}^{t}\left[\dot{x}\left(t_{2}\right)-\underline{a}_{0} \frac{\delta}{2}\left(\tau-t_{2}\right)\right] \mathrm{d} \tau \\
& =\dot{x}\left(t_{2}\right)\left(t-t_{2}\right)-\frac{\underline{a}_{0} \delta}{4}\left(t-t_{2}\right)^{2} \quad \forall t \geq t_{2} .
\end{aligned}
$$

Since the right-hand side of this inequality tends to $-\infty$ as $t \rightarrow \infty$ and the left-hand side is bounded, this is a contradiction. The case $x(t) \leq \delta$ for all $t \geq t_{1}$ yields a contradiction in a similar manner. Thus $V(t) \rightarrow 0$ as $t \rightarrow \infty$, proving that $x(t) \rightarrow 0$, i.e. attractivity, and the proof of the theorem is complete.

ExAmPle 2.3. Consider the equation

$$
\ddot{x}+\dot{x}+a_{0}(t) x=0,
$$

where $a_{0}$ denotes a differentiable approximation of $\hat{a}_{0}$ defined, for the sequence $t_{n}=$ $\frac{n(n-1)}{2}, n \geq 2$, as follows:

$$
\hat{a}_{0}(t)= \begin{cases}t+1, & t \in[0,1], \\ -\frac{n}{n^{2}-1} t+\frac{n^{2}}{2(n+1)}+2, & t \in\left[t_{n}, t_{n}+n-\frac{1}{n}\right], \\ n t+2-\frac{n^{2}(n+1)}{2}, & t \in\left[t_{n}+n-\frac{1}{n}, t_{n+1}\right] .\end{cases}
$$

See Figure 1. There certainly exists an approximation $a_{0}$ of $\hat{a}_{0}$ such that the assumptions (2.1)-(2.3) are satisfied and so by Theorem 2.1 the zero solution of (2.4) is attractive and uniformly stable. However, the approximation $a_{0}$ has unbounded derivative, and hence Theorem 1.8 cannot be applied.

The next theorem deals with time-dependent, sign indefinite but bounded $a_{1}(\cdot)$ (including the case of small damping; see Hatvani's Theorem 1.6); moreover, the coefficient $a_{0}(\cdot)$ is only assumed to be bounded but not necessarily bounded away from 0 . 


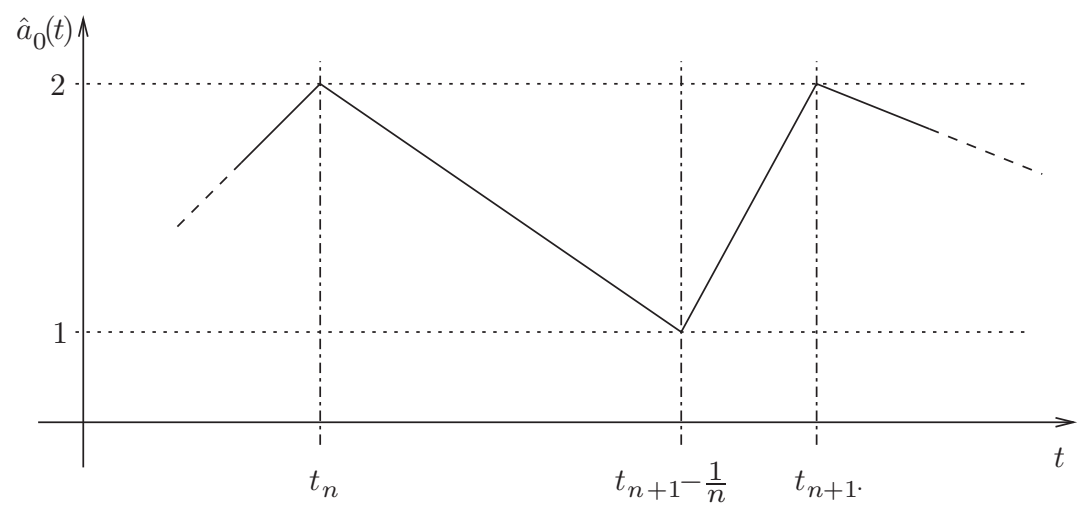

Fig. 1. The essential derivative of $\hat{a}_{0}$ is unbounded.

THEOREM 2.4. Suppose that the coefficients of (1.1) satisfy the following:

$$
\begin{aligned}
& \int_{0}^{t} a_{0}(\tau) \mathrm{d} \tau \rightarrow \infty \quad \text { as } t \rightarrow \infty, \\
\exists M, \bar{a}_{0}>0 \forall t \geq 0: & 0<a_{0}(t) \leq \bar{a}_{0}, \\
\exists M, \bar{a}_{1}>0 \forall t \geq 0: & \left|a_{1}(t)\right| \leq \bar{a}_{1}, \quad \text { and } \quad\left|\dot{a}_{1}(t)\right|<M\left|a_{0}(t)\right|, \\
\exists k>0 \forall t \geq 0: & 0<k a_{0}(t)^{2} \leq \dot{a}_{0}(t)+2 a_{0}(t) a_{1}(t) .
\end{aligned}
$$

Then the zero solution of (1.1) is asymptotically stable.

REMARK 2.5. If the rigidity coefficient $a_{0}(\cdot)=a_{0}$ is constant and positive, then the assumptions of Theorem 2.4 specialize to

$$
\forall t \geq 0: \frac{k a_{0}}{2} \leq a_{1}(t) \leq \bar{a}_{1} \quad \text { and } \quad a_{0}\left|\dot{a}_{1}(t)\right|<M .
$$

Proof of Theorem 2.4. Set

$$
\begin{array}{rlrl}
b & :=\min \left\{\frac{1}{2 \bar{a}_{1}}, \frac{k}{M+4+4 b^{2} \bar{a}_{0}+\bar{a}_{1} k}\right\}, & \\
c(t):=\frac{2 b^{2} a_{0}(t)-b a_{1}(t)+1}{a_{0}(t)} & \forall t \geq 0, \\
d(t):=-2 b-\dot{c}(t)-2 b a_{0}(t) c(t)+2 a_{1}(t)+1 & \forall t \geq 0 .
\end{array}
$$

Note that

$$
c(t)-b^{2} \geq b^{2}+\frac{1}{2 a_{0}(t)} \quad \forall t \geq 0
$$

and since

it follows that

$$
b \leq \frac{k}{M+4+4 b^{2} \bar{a}_{0}+\bar{a}_{1} k},
$$

$$
\left(1-b \bar{a}_{1}\right) k+b\left[-M-4-4 b^{2} \bar{a}_{0}\right] \geq 0,
$$


and therefore

$$
\begin{aligned}
d(t)=\left(1-b \bar{a}_{1}(t)\right) & \frac{\dot{a}_{0}(t)+2 a_{0}(t) a_{1}(t)}{a_{0}(t)^{2}} \\
& +b\left(\frac{\dot{a}_{1}(t)}{a_{0}(t)}-4\right)-6 b^{2} a_{1}(t)-4 b^{2} a_{0}(t) \geq 0 \quad \forall t \geq 0 .
\end{aligned}
$$

The derivative of

$$
W(t):=(x(t)+b \dot{x}(t))^{2}+2\left(c(t)-b^{2}\right) \dot{x}(t)^{2} \quad \forall t \geq 0,
$$

along the solution of (1.1) yields

$$
\dot{W}(t)=-2 b a_{0}(t) W(t)-d(t) \dot{x}(t)^{2} \leq-2 b a_{0}(t) W(t) \leq 0 \quad \forall t \geq 0
$$

and so, by integration,

$$
W(t) \leq \exp \left(-2 b \int_{0}^{t} a_{0}(\tau) d \tau\right) W\left(t_{0}\right) \quad \forall t \geq 0 .
$$

Equation (2.11) shows stability of the zero solution. Moreover, in view of (2.5), we arrive at $\lim _{t \rightarrow \infty} W(t)=0$, and therefore, $x(t)+b \dot{x}(t) \rightarrow 0$, as well as $b^{2} \dot{x}^{2}(t)<\left(c-b^{2}\right) \dot{x}^{2}(t) \rightarrow 0$ as $t \rightarrow \infty$. Finally, this implies that $\dot{x}(t) \rightarrow 0$ and thus also that $x(t) \rightarrow 0$ as $t \rightarrow \infty$, which proves attractivity of the zero solution of (1.1), and by Remark 1.2(b) it also proves asymptotic stability, and hence the proof of the theorem is complete.

EXAMPLE 2.6. It readily follows from Theorem 2.4 that the zero solution of the equation

$$
\ddot{x}+\frac{1}{1+t} \dot{x}+\frac{1}{1+t} x=0
$$

is asymptotically stable.

In the following two theorems we use a time-dependent transformation to transform equation (1.1) into $\ddot{z}+b(t) z=0$. We distinguish the cases $b(t) \geq 0$ and $b(t) \leq 0$, for all $t \geq 0$. In both cases this method allows us to treat unbounded coefficients.

THEOREM 2.7. Suppose that the coefficients of equation (1.1) satisfy

$$
\begin{aligned}
& b(t):=a_{0}(t)-\frac{1}{2} \dot{a}_{1}(t)-\frac{1}{4} a_{1}(t)^{2}>0 \quad \forall t \geq 0, \\
& \lim _{t \rightarrow \infty} a_{1}(t) e^{-\frac{1}{2} \int_{0}^{t}\left(a_{1}(\tau)-g(\tau)\right) \mathrm{d} \tau}=0, \\
& \lim _{t \rightarrow \infty} \sqrt{b(t)} e^{-\frac{1}{2} \int_{0}^{t}\left(a_{1}(\tau)-g(\tau)\right) \mathrm{d} \tau}=0, \\
& \int_{0}^{t}\left[a_{1}(\tau)-g(\tau)\right] \mathrm{d} \tau \rightarrow \infty \quad \text { as } t \rightarrow \infty,
\end{aligned}
$$

where, for all $t \geq 0, g(t):=\max \{0,-\dot{b}(t) / b(t)\}$. Then the zero solution of equation (1.1) is asymptotically stable.

REMARK 2.8. By Remark 1.2(c) it suffices to check whether condition (2.12) holds only for all $t \geq t_{0}$ for some $t_{0} \geq 0$. 
Proof of Theorem 2.7. Let $\left(x^{0}, x^{1}\right) \in \mathbb{R} \times \mathbb{R}$ and let $x(\cdot) \equiv x\left(\cdot ; 0, x^{0}, x^{1}\right)$ be the solution to (1.1). The transformation

$$
z(t)=x(t) e^{\frac{1}{2} \int_{0}^{t} a_{1}(\tau) d \tau} \quad \forall t \geq 0,
$$

yields that (1.1) is equivalent to

$$
\ddot{z}+b(t) z=0 \quad \forall t \geq 0,
$$

with $z \not \equiv 0$. The derivative of

$$
V(t)=\sqrt{z(t)^{2}+\dot{z}(t)^{2} / b(t)} \quad \forall t \geq 0,
$$

along the solution of (2.16) satisfies

$$
2 \dot{V}(t) V(t)=-\frac{\dot{b}(t)}{b(t)} \frac{\dot{z}(t)^{2}}{b(t)} \leq g(t)\left[V(t)^{2}-z(t)^{2}\right] \leq g(t) V(t)^{2} \quad \forall t \geq 0,
$$

and hence integration gives

$$
V(t) \leq e^{\frac{1}{2} \int_{0}^{t} g(\tau) d \tau} V(0) \quad \forall t \geq 0,
$$

whence

$$
|x(t)|=|z(t)| e^{-\frac{1}{2} \int_{0}^{t} a_{1}(\tau) d \tau} \leq e^{\frac{1}{2} \int_{0}^{t}\left(g(\tau)-a_{1}(\tau)\right) d \tau} V(0) \quad \forall t \geq 0,
$$

and so, invoking (2.15), we have $\lim _{t \rightarrow \infty} x(t)=0$. Since

$$
\begin{aligned}
|\dot{x}(t)| & \leq|\dot{z}(t)| e^{-\frac{1}{2} \int_{0}^{t} a_{1}(\tau) d \tau}+\frac{1}{2}\left|a_{1}(t)\right||z(t)| e^{-\frac{1}{2} \int_{0}^{t} a_{1}(\tau) d \tau} \\
& \leq\left(\sqrt{b(t)}+\frac{1}{2}\left|a_{1}(t)\right|\right) V(0) e^{\frac{1}{2} \int_{0}^{t}\left(g(\tau)-a_{1}(\tau)\right) d \tau} \quad \forall t \geq 0,
\end{aligned}
$$

an application of (2.13), (2.14) gives $\lim _{t \rightarrow \infty} \dot{x}(t)=0$. Together with Remark $1.2(\mathrm{c})$, this proves attractivity of the zero solution of (1.1), and by Remark 1.2(b) it also proves asymptotic stability. This completes the proof of the theorem.

Corollary 2.9. Suppose the coefficients of (1.1) satisfy $a_{1}(\cdot) \equiv a_{1}$ constant and $\dot{a}_{0}(t) \geq$ 0 for all $t \geq 0$. Then $g(\cdot) \equiv 0$, and (2.13), (2.15) in Theorem 2.7 are trivially fulfilled, and a sufficient condition for attractivity of the zero solution becomes

$$
b(t)=a_{0}(t)-\frac{1}{4} a_{1}^{2}>0 \forall t \geq 0 \quad \text { and } \quad \lim _{t \rightarrow \infty} b(t) e^{-a_{1} t}=0 .
$$

Thus, we may choose, for $0<\lambda<a_{1}$, a non-decreasing function $a_{0}(\cdot)$ with $\frac{a_{1}^{2}}{4} \leq a_{0}(t) \leq$ $e^{\lambda t}$ for all $t \geq 0$. In this case, the zero solution of equation (1.1) is asymptotically stable.

ExAmple 2.10. Consider, for $\alpha \in(0,1)$, the equation

$$
\ddot{x}+2(t+1)^{-\alpha} \dot{x}+x=0 .
$$

Then a straightforward calculation yields the existence of some $t_{0} \geq 0$ such that, for any $t \geq t_{0}$

$$
\begin{aligned}
1+\alpha \geq b(t) & =1+\alpha(t+1)^{-\alpha-1}-(t+1)^{-2 \alpha}>0 \\
\dot{b}(t) & =-\alpha(\alpha+1)(t+1)^{-\alpha-2}+2 \alpha(t+1)^{-2 \alpha-1} \\
& =\alpha(t+1)^{-\alpha-2}\left(2(t+1)^{1-\alpha}-\alpha-1\right)>0 .
\end{aligned}
$$


In this case $g(t)=0$ for $t \geq t_{0}$ and therefore, for any $t \geq t_{0}$,

$$
\begin{aligned}
\int_{0}^{t}\left(a_{1}(\tau)-g(\tau)\right) d \tau & =\int_{0}^{t_{0}}\left(a_{1}(\tau)-g(\tau)\right) d \tau+\int_{t_{0}}^{t}\left(a_{1}(\tau)-g(\tau)\right) d \tau \\
& =\int_{0}^{t_{0}}\left(a_{1}(\tau)-g(\tau)\right) d \tau+2 \int_{t_{0}}^{t}(\tau+1)^{-\alpha} d \tau \\
& =\int_{0}^{t_{0}}\left(a_{1}(\tau)-g(\tau)\right) d \tau+\frac{2}{1-\alpha}\left((t+1)^{1-\alpha}-\left(t_{0}+1\right)^{1-\alpha}\right) .
\end{aligned}
$$

Hence, the suppositions of Theorem 2.7 are satisfied, and the zero solution of equation (2.18) is asymptotically stable. Note that the criterion of Hatvani [5] cannot be applied to this equation if $\alpha>\frac{1}{3}$.

In the following theorem we treat the case $b(t)<0$ for all $t \geq 0$.

TheOrem 2.11. Suppose that the coefficients in equation (1.1) satisfy

$$
\begin{aligned}
& \beta(t):=\frac{1}{4} a_{1}(t)^{2}+\frac{1}{2} \dot{a}_{1}(t)-a_{0}(t)>0 \quad \forall t \geq 0, \\
& \lim _{t \rightarrow \infty} a_{1}(t) \sqrt{\beta(t)+1} e^{\int_{0}^{t}\left(h(\tau)-\frac{1}{2} a_{1}(\tau)\right) \mathrm{d} \tau}=0, \\
& \lim _{t \rightarrow \infty} \sqrt{\beta(t)+1} e^{\int_{0}^{t}\left(h(\tau)-\frac{1}{2} a_{1}(\tau)\right) d \tau}=0,
\end{aligned}
$$

where,

$$
h(t):=\left|\frac{\dot{\beta}(t)}{4 \beta(t)}\right|+\sqrt{\beta(t)+\left(\frac{\dot{\beta}(t)}{4 \beta(t)}\right)^{2}} \quad \text { for all } t \geq 0 .
$$

Then the zero solution of (1.1) is asymptotically stable.

REMARK 2.12. By Remark 1.2(c) it suffices to check whether condition (2.19) holds only for all $t \geq t_{0}$ for some $t_{0} \geq 0$.

Proof of Theorem 2.11. Using the same notation as in the proof of Theorem 2.7, we see that $\beta=-b$, and so equation (1.1) is equivalent to $\ddot{z}-\beta(t) z=0$, which is equivalent, under $y=(z, \dot{z})^{T}$, to its first order formulation

$$
\dot{y}=\left[\begin{array}{cc}
0 & 1 \\
\beta(t) & 0
\end{array}\right] y
$$

Applying the transformation

$$
y=T(t) u=\left[\begin{array}{cc}
1 & 1 \\
\sqrt{\beta(t)} & -\sqrt{\beta(t)}
\end{array}\right] u
$$

yields

$$
\dot{u}=B(t) u, \quad \text { where } B=T^{-1} A T-T^{-1} \dot{T}=\left[\begin{array}{cc}
\sqrt{\beta}-\dot{\beta} / 4 \beta & \dot{\beta} / 4 \beta \\
\dot{\beta} / 4 \beta & -\sqrt{\beta}-\dot{\beta} / 4 \beta
\end{array}\right] .
$$

Note that if $\lambda(t)$ denotes the eigenvalue of $B(t)$ with biggest absolute value, then $h(t)=$ $|\lambda(t)|$ and, invoking that $B(t)$ is symmetric,

$$
\frac{\mathrm{d}}{\mathrm{d} t}\|u(t)\| \leq\|\dot{u}(t)\|=\|B(t) u\| \leq h(t)\|u(t)\| \quad \text { for almost all } t \geq 0,
$$


and so

$$
\begin{aligned}
& \|y(t)\|=\|T(t) u(t)\| \leq\|T(t)\|\|u(t)\| \\
& \leq \sqrt{2+2 \beta(t)}\|u(0)\| e^{\int_{0}^{t} h(\tau) \mathrm{d} \tau} \quad \text { for all } t \geq 0 .
\end{aligned}
$$

Furthermore,

$$
\begin{aligned}
|x(t)| & \leq|z(t)| e^{-\frac{1}{2} \int_{0}^{t} a_{1}(\tau) \mathrm{d} \tau} \\
& \leq\|y(t)\| e^{-\frac{1}{2} \int_{0}^{t} a_{1}(\tau) d \tau} \\
& \leq \sqrt{2-2 b(t)}\|u(0)\| e^{\int_{0}^{t}\left[h(\tau)-\frac{1}{2} a_{1}(\tau)\right] \mathrm{d} \tau} \quad \text { for all } t \geq 0
\end{aligned}
$$

and

$$
\begin{aligned}
& |\dot{x}(t)| \leq|\dot{z}(t)| e^{-\frac{1}{2} \int_{0}^{t} a_{1}(\tau) \mathrm{d} \tau}+\frac{1}{2}\left|a_{1}(t)\right||z(t)| e^{-\frac{1}{2} \int_{0}^{t} a_{1}(\tau) \mathrm{d} \tau} \\
& \leq\left[1+\frac{1}{2}\left|a_{1}(t)\right|\right]\|y(t)\| e^{-\frac{1}{2} \int_{0}^{t} a_{1}(\tau) \mathrm{d} \tau} \\
& \leq\left[1+\frac{1}{2}\left|a_{1}(t)\right|\right] \sqrt{2+2 \beta(t)}\|u(0)\| e^{\int_{0}^{t}\left[h(\tau)-\frac{1}{2} a_{1}(\tau)\right] \mathrm{d} \tau} \quad \text { for all } t \geq 0 .
\end{aligned}
$$

The conclusion now follows from the assumptions.

EXAMPLE 2.13. The unbounded coefficients of the differential equation

$$
\ddot{x}+t \dot{x}+(t-1) x=0
$$

satisfy the assumptions of Theorem 2.11, and therefore its zero solution is asymptotically stable.

The following two theorems are based on a more geometric point of view. In Theorem 2.14 we construct a polygon which is invariant under the dynamics forward in time and give conditions for the stability of system (1.2) or equivalently equation (1.1). In Theorem 2.15 we strengthen the hypothesis and provide geometric conditions for asymptotic stability. For both theorems, we consider, for $r>0$, the compact set $S^{r}$ by the boundary given by the polygon

$$
\begin{aligned}
& s_{1}^{r}:=\left\{\left(y_{1}, y_{2}\right) \mid y_{1}=r, y_{2} \in[-r, 0]\right\}, \\
& s_{2}^{r}:=\left\{\left(y_{1}, y_{2}\right) \mid y_{1} \in[0, r], y_{2}=-r\right\}, \\
& s_{3}^{r}:=\left\{\left(y_{1}, y_{2}\right) \mid y_{1} \in[-r, 0], y_{2}=-r-y_{1}\right\},
\end{aligned}
$$

the point symmetric lines

$$
\hat{s}_{i}^{r}:=\left\{\left(-y_{1},-y_{2}\right) \mid\left(y_{1}, y_{2}\right) \in s_{i}^{r}\right\} \quad \text { for } i=1,2,3,
$$

and $0 \in \operatorname{int} S^{r}$.

THEOREM 2.14. Suppose the coefficients of the equation (1.1) satisfy

$$
\begin{array}{ll}
\forall t \geq 0: & 0<a_{0}(t) \leq a_{1}(t), \\
\forall t \geq 0: & 1 \leq a_{1}(t) .
\end{array}
$$

Then the zero solution of equation (1.1) is uniformly stable. 


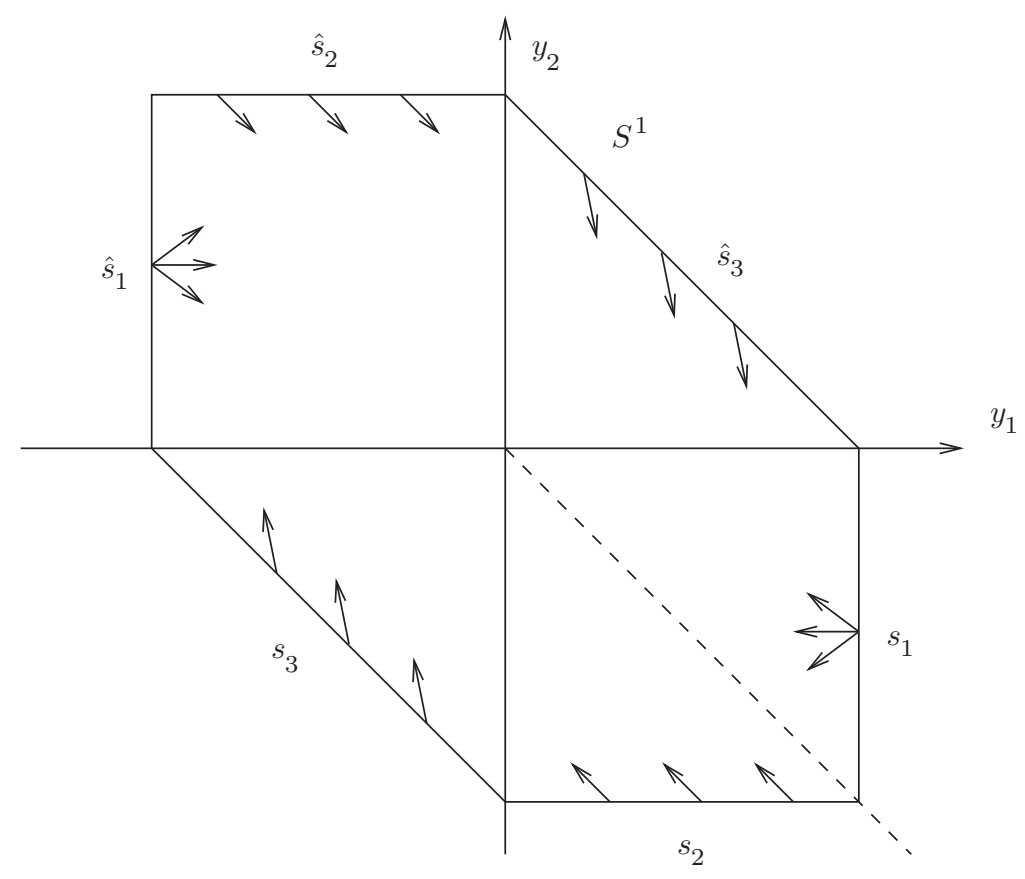

FIG. 2. The vector field points into the forward invariant polygon $S^{1}$.

Proof of Theorem 2.14. First, we show that $S^{1}$ is forward invariant, i.e. for any initial data $\left(y_{1}^{0}, y_{2}^{0}\right) \in S^{1}$ it follows that the unique solution of (1.2) satisfies, for all $t \geq 0$, $\left(y_{1}(t), y_{2}(t)\right) \in S^{1}$. Invoking (2.22) and (2.23), the following implications show that the vector field given by the right-hand side of (1.2) points into $S^{1}$ along the polygon $s_{1} \cup s_{2} \cup s_{3}$. We omit the superscript "r" for $r=1$ and the argument $t$ in the following (see also Figure 2):

$$
\begin{array}{lll}
\left(y_{1}, y_{2}\right)=(1,0) & \Longrightarrow & \dot{y}_{1}=0, \dot{y}_{2}=-a_{0}<0, \\
\left(y_{1}, y_{2}\right) \in s_{1} & \Longrightarrow & \dot{y}_{1}=y_{2} \leq 0, \dot{y}_{2}=-a_{0}-a_{1} y_{2} \in \mathbb{R}, \\
\left(y_{1}, y_{2}\right)=(1,-1) & \Longrightarrow & \dot{y}_{1}=-1, \dot{y}_{2}=-a_{0}+a_{1} \geq 0, \\
\left(y_{1}, y_{2}\right) \in s_{2} & \Longrightarrow & \dot{y}_{1}=-1, \dot{y}_{2}=-a_{0} y_{1}+a_{1} \geq-a_{0}+a_{1} \geq 0, \\
\left(y_{1}, y_{2}\right)=(0,-1) & \Longrightarrow & \left(\dot{y}_{1}, \dot{y}_{2}\right)=\left(-1, a_{1}\right) \text { with } a_{1} \geq 1, \\
\left(y_{1}, y_{2}\right)=(-1,0) & \Longrightarrow & \left(\dot{y}_{1}, \dot{y}_{2}\right)=\left(0, a_{0}\right) \text { with } a_{0}>0, \\
\left(y_{1}, y_{2}\right) \in s_{3} & \Longrightarrow & \dot{y}_{1}=-1-y_{1}, \dot{y}_{2} \geq a_{0}-a_{1}\left[-1-y_{0}\right] .
\end{array}
$$

By point symmetry, the vector field of (1.2) is also pointing inwards along the polygon $\hat{s}_{1} \cup \hat{s}_{2} \cup \hat{s}_{3}$. Therefore, $S^{1}$ is forward invariant and since (1.2) is linear, it follows that the zero solution of (1.2) is uniformly stable. This completes the proof of the theorem.

TheOrEm 2.15. Suppose there exist $\varepsilon, M>0$ such that the coefficients of equation (1.1) satisfy

$$
\begin{array}{ll}
\forall t \geq 0: & 0<\varepsilon \leq a_{0}(t) \leq a_{1}(t) \leq M, \\
\forall t \geq 0: & 1+\varepsilon \leq a_{1}(t) .
\end{array}
$$


Then the zero solution of equation (1.1) is asymptotically stable.

Proof of Theorem 2.15. By Theorem 2.14 and Remark 1.2(b), it suffices to prove attractivity of the zero solution. We proceed in two steps.

(i) If $y(\cdot)$ crosses the $y_{1}$-axis only finitely many times, then there exists $t_{0} \geq 0$ such that, for all $t \geq t_{0}$,

(a) $\left(y_{1}(t), y_{2}(t)\right) \in S^{r} \cap\left(\mathbb{R} \times \mathbb{R}_{\geq 0}\right)$

or

(b) $\left(y_{1}(t), y_{2}(t)\right) \in S^{r} \cap\left(\mathbb{R} \times \mathbb{R}_{\leq 0}\right)$.

We prove that in case (b) the claim of the theorem follows; case (a) is proved analogously and omitted for brevity.

Since $\dot{y}_{1}(t)=y_{2}(t) \leq 0$ for all $t \geq t_{0}$ and $y_{1}$ is bounded, there exists $\widetilde{M}>0$ such that

$$
0 \leq \int_{t_{0}}^{t}-y_{2}(\tau) d \tau=y_{1}\left(t_{0}\right)-y_{1}(t) \leq \widetilde{M} \quad \forall t \geq t_{0} .
$$

Therefore, $y_{2} \in L^{1}$, and applying boundedness of $y_{1}$ and $y_{2}$ together with (2.24) to

$$
\left|\dot{y}_{2}(t)\right|=\left|a_{0}(t) y_{1}(t)+a_{1}(t) y_{2}(t)\right| \leq M\left(\left|y_{1}(t)\right|+\left|y_{2}(t)\right|\right)
$$

gives $\dot{y}_{2} \in L^{\infty}$. So we may apply Barbălat's lemma (see [2]) to conclude that $y_{2}(t) \rightarrow 0$ as $t \rightarrow \infty$.

By monotonicity of $y_{1}$, there exists $y_{1}^{*} \in \mathbb{R}$ such that $y_{1}(t) \rightarrow y_{1}^{*}$ as $t \rightarrow \infty$. Applying (2.24) again gives

$$
\exists t_{1} \geq t_{0} \forall t \geq t_{1}: \frac{\varepsilon}{2}\left|y_{1}^{*}\right|=\left|\dot{y}_{2}\right| \leq 2 M\left|y_{1}^{*}\right| .
$$

Since $y_{2}(t) \rightarrow 0$ as $t \rightarrow \infty$, it follows that $y_{1}^{*}=0$.

(ii) Since the vector field along $(0, r) \times\{0\}$ is $\dot{y}_{2}=-a_{1} y_{1}<0, \dot{y}_{1}=0$, and along $(-r, 0) \times\{0\}$ is $\dot{y}_{2}=-a_{1} y_{1}>0, \dot{y}_{1}=0$, it remains to consider the case that there exists a sequence $\left(t_{n}\right)_{n \in \mathbb{N}}$, with

$$
t_{n}<t_{n+1} \quad \text { and } \quad y_{2}\left(t_{n}\right)=0, y_{1}\left(t_{n}\right)>0 \quad \forall n \in \mathbb{N} .
$$

In the proof of Theorem 2.14 we have shown that $S^{r}, r>0$, is positively invariant. Observe that, by (2.24) and (2.25), the vector field along the segment

$$
L:=\left\{\left(y_{1}, y_{2}\right) \in(-r /(1+\varepsilon), 0) \times(0,-r) \mid y_{2}=-(1+\varepsilon) y_{1}-r\right\}
$$

satisfies

$$
\left(\dot{y}_{1}, \dot{y}_{2}\right) \in(-r, 0) \times\left(\frac{a_{0}}{1+\varepsilon}, a_{1}\right)
$$

and consequently a solution starting in the polygon

$$
L \cup\left(\left[\frac{-r}{1+\varepsilon}, r\right] \times\{0\}\right) \cup(\{r\} \times[-r, 0]) \cup([0, r] \times\{-r\})
$$

can leave only through the segment $\left[\frac{-r}{1+\varepsilon}, 0\right] \times\{0\}$ (see also Figure 3 ). We may choose $t_{0} \geq 0$ such that $y_{2}\left(t_{0}\right)=0$ and $r:=y_{1}\left(t_{0}\right)$. By the above observations, we may also choose a sequence $\left(t_{n}\right)_{n \in \mathbb{N}}$ such that $y_{2}\left(t_{n}\right)=0$ and

$$
0<y_{1}\left(t_{n+1}\right) \leq \frac{1}{1+\varepsilon} y_{1}\left(t_{n}\right) .
$$




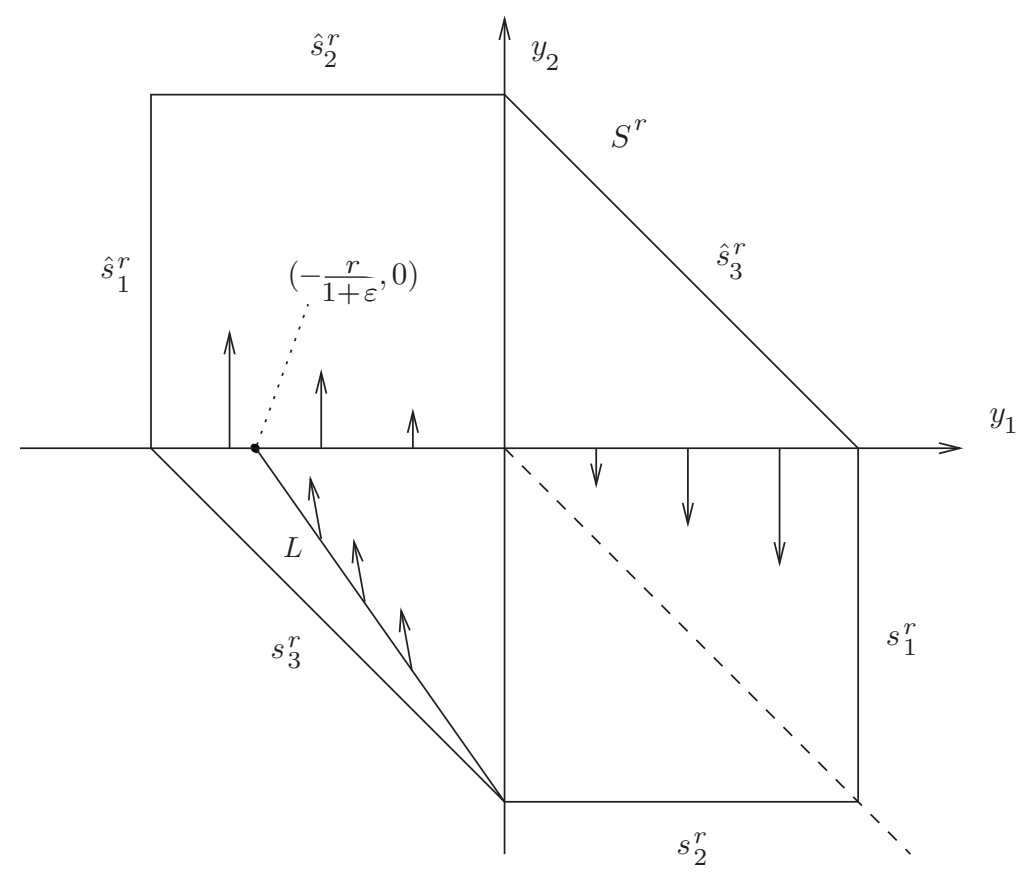

FIG. 3. Solutions in $S^{r}$ starting in the $4^{\text {th }}$ quadrant do not cross the line $L$.

Together with the fact that

$$
\left|y_{2}(t)\right| \leq\left|y_{1}\left(t_{n}\right)\right| \quad \text { for } t_{n} \leq t \leq t_{n+1}
$$

(see also Figure 3) this implies that $y(t) \rightarrow 0$ as $t \rightarrow \infty$, and the proof of the theorem is complete.

Condition (2.25) ensures that each time a solution completes a rotation around the origin, the norm is reduced by a factor less than 1 . Whereas this condition might not be necessary for asymptotic stability, condition (2.24) cannot be omitted, as the following examples show.

ExAmple 2.16. The condition $0<\varepsilon<a_{0}(t)$ for all $t \geq 0$ in Theorem 2.15 cannot be omitted, as the equation

$$
\ddot{x}+\left(2+e^{-t}\right) \dot{x}+e^{-t} x=0
$$

shows. It has the solution $t \mapsto x(t)=1+e^{-t}$ which does not converge to 0 for $t \rightarrow \infty$.

Example 2.17. The condition $\left\|a_{1}\right\|_{\infty} \in \mathbb{R}$ in Theorem 2.15 cannot be omitted, as the equation

$$
\ddot{x}+\left(2+e^{t}\right) \dot{x}+x=0
$$

shows (cf. equation (1.5) with $a_{0}=1$ ). It has the solution $t \mapsto x(t)=1+e^{-t}$, which does not converge to 0 for $t \rightarrow \infty$. 


\section{REFERENCES}

[1] Z. Artstein and E.F. Infante, On the asymptotic stability of oscillators with unbounded damping. Quart. Appl. Math. 34 (1976/77), no. 2, 195-199. MR0466789 (57:6665)

[2] I. Barbălat, Systèmes d'équations différentielles d'oscillations nonlinéaires, Revue de Mathématiques Pures et Appliquées, Bucharest IV (1959), 267-270. MR0111896(22:2754b)

[3] C.E. Desoer, Slowly varying system $\dot{x}=A(t) x$. IEEE Trans. Automatic Control 14 (1969), 780-781. MR0276562 (43:2306)

[4] L. Dieci and E. Van Vleck, Lyapunov and other spectra: a survey. Collected lectures on the preservation of stability under discretization (Fort Collins, CO, 2001), 197-218, SIAM, Philadelphia, PA, 2002. MR 2026670

[5] L. Hatvani, Integral conditions on the asymptotic stability for the damped linear oscillator with small damping. Proc. Amer. Math. Soc. 124 (1996), 415-422. MR1317039 (96d:34062)

[6] L. Hatvani, T. Krisztin, and V. Totik, A necessary and sufficient condition for the asymptotic stability of the damped oscillator. J. Differential Equations 119 (1995), 209-223.

[7] F.C. Hoppenstaed, Singular perturbations on the infinite interval. Trans. Am. Math. Soc. 123 (1966), 521-535. MR0194693 (33:2900)

[8] G.R. Hovhannisyan, Asymptotic stability for second-order differential equations with complex coefficients. Electron. J. Differential Equations 85 (2004), 20 pp. MR2075424 (2005f:34141)

[9] A.O. Ignatyev, Stability of a linear oscillator with variable parameters. Electron. J. Differential Equations 17 (1997), 6 pp. MR1476064 (98i:34076)

[10] J.J. Levin and J.A. Nohel, Global asymptotic stability for nonlinear systems of differential equations and applications to reactor dynamics. Arch. Rational Mech. Anal. 5 (1960), 194-211. MR0119524 $(22: 10285)$

[11] P. Pucci and J. Serrin, Precise damping conditions for global asymptotic stability for nonlinear second order systems. Acta Math. 170 (1993), no. 2, 275-307. MR.1226530 (94i:34103)

[12] P. Pucci and J. Serrin, Precise damping conditions for global asymptotic stability for nonlinear second order systems. II. J. Differential Equations 113 (1994), no. 2, 505-534. MR1297668 (95m:34088)

[13] P. Pucci and J. Serrin, Asymptotic stability for intermittently controlled nonlinear oscillators. SIAM J. Math. Anal. 25 (1994), no. 3, 815-835. MR1271312 (95c:34092)

[14] H.H. Rosenbrock, The stability of linear time-dependent control systems. J. Electronics Control 15 (1963), 73-80. MR0159085 (28:2303)

[15] W.J. Rugh (1996), Linear System Theory 2nd ed., Prentice-Hall, Englewood Cliffs, New Jersey. MR 1211190 (94b:93002)

[16] R.J. Sacker and G.R. Sell, A Spectral Theory for Linear Differential Systems. J. Differential Equations 27 (1978), 320-358. MR0501182 (58:18604)

[17] S. Siegmund, Dichotomy spectrum for nonautonomous differential equations. J. Dynam. Differential Equations 14 (2002), no. 1, 243-258. MR1878650(2002j:34082)

[18] R.A. Smith, Asymptotic stability of $x^{\prime \prime}+a(t) x^{\prime}+x=0$. Quart. J. Math. Oxford Ser. (2) 12 (1961), 123-126. MR0124582 (23:A1894)

[19] V. Solo, On the stability of slowly time-varying linear systems. Math. Control Signals Systems 7 (1994), no. 4, 331-350. MR.1359034 (96k:34108) 